\title{
Prevalencia de disfunción sexual en mujeres climatéricas
}

\author{
Rodrigo Figueroa Ja, D aniela Jara Aa, Andrea Fuenzalida Pa, \\ Mónica del Prado A ${ }^{\mathrm{a}}$, Daniel Flores ${ }^{1}$, Juan E Blumel${ }^{2}$. \\ Prevalence of sexual dysfunction \\ among climacteric women
}

Background: The Female Sexual Function Index (FSFI), is a scale designed to evaluate sexuality and diagnose the presence of sexual dysfunction in women. Aim: To apply the FSFI to climacteric women. Patients and methods: The FSFI was applied to 370 healthy women aged between 40 and 59 years old ( $49 \pm 6$ years) that accompanied patients to public health services in Santiago. Results: Fifty six percent of women were married, $44 \%$ were postmenopausal, 6\% used hormone replacement therapy, 67\% were sexually active, and sexual dysfunction was present in 57\% of them. Thirty two percent of women aged between 40 and 44 years and 65\% of women aged between 55 and 59 years, had sexual dysfunction $(p<0.01)$. In a logistic regression model, the risk of sexual dysfunction increased among women that perceive having health problems (Odds ratio (OR) 3.9; 95\% confidence intervals (95\% CI): 1.1-13.8), women older than 48 years (OR 1.9; 95\% CI: 1.1-3.4) and women that gave birth to two or more children (OR 1.8; 95\% CI: 1.0-3.1). Conclusions: Climateric women have high prevalence of sexual dysfunction. Age is its main risk factor (Rev Méd Chile 2009; 137: 345-50). (Key words: Climacteric; Menopause; Sexual dysfunction, physiological)

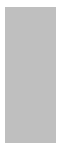

\footnotetext{
Recibido el 22 de abril, 2008. Aceptado el 5 de diciembre, 2008.

${ }^{1}$ Departamento de Gineco-obstetricia, Escuela de Medicina, Universidad Diego Portales, Santiago, Chile. ${ }^{2}$ Hospital Barros Luco-Trudeau, Santiago, Chile.

anterno de Medicina, Universidad Diego Portales. Santiago, Chile.
}

$\mathrm{L}^{2}$ a sexualidad es un aspecto central de la calidad de vida de la mujer y es reflejo de su bienestar físico, psicológico y social ${ }^{1}$. El climaterio impacta negativamente en la sexualidad ${ }^{2}$, pudiendo variar entre las diferentes personas, e incluso entre diferentes países, influenciado por factores biológicos y socioculturales ${ }^{3,4}$.

La sexualidad humana es un fenómeno complejo, comprende procesos tanto psicológicos como orgánicos y su comportamiento ha variado

Correspondencia a: Rodrigo Figueroa J. Casilla $\mathrm{n}^{\circ} 78$, San Bernardo. Santiago de Chile. Fax: 8847138.

E mail: rodrigo.figueroa@gmail.com en el tiempo. Por este motivo resulta tan complejo su estudio, que sumado a los problemas metodológicos que han presentado la mayoría de los estudios que investigan el tema, resulta en una dificultad para interpretar y comparar los resultados publicados sobre esta área de la conducta humana ${ }^{5}$

Rosen y cols, basados en el International Consensus Development Conference on Female Sexual Dysfunctions ${ }^{6}$ desarrollaron un test con propiedades psicométricas, capaz de evaluar la función sexual en mujeres en sus diversos dominios: deseo, excitación, orgasmo, dolor, lubricación y satisfacción. Lo denominaron "Índice de Función Sexual Femenina" (IFSF) ${ }^{7}$, el cual ha 
demostrado altos niveles de confiabilidad, consistencia interna, aplicabilidad en un amplio rango de edad, y ha sido validado clínicamente en mujeres chilenas de 20 a 59 años 8 .

Los estudios existentes en nuestro país sobre sexualidad femenina son escasos, y los realizados en mujeres climatéricas se centran en grupos con patologías específicas o usuarias de alguna terapia en particular. No existen estudios que evalúen la prevalencia de disfunción sexual en mujeres sanas de edad media mediante el IFSF.

El objetivo de este estudio es aplicar el test desarrollado por Rosen en mujeres en la etapa del climaterio y evaluar la presencia de disfunción sexual y sus variables asociadas.

\section{PACIENTES Y MÉTODO}

Estudio descriptivo de corte transversal, en el período comprendido entre agosto y noviembre de 2007. La muestra estuvo constituida por mujeres de 40 a 59 años, que acudieron como acompañantes a centros de salud de la ciudad de Santiago (Hospital San José, Hospital Dipreca, Hospital de Urgencias Asistencia Pública). Para el cálculo del tamaño muestral se utilizó el programa estadístico Epi-Info 6.04; con una frecuencia esperada de trastornos de sexualidad de $51 \% 5$, una población estimada en la ciudad de Santiago de 600.000 mujeres entre 40 a 59 años, un error máximo aceptable de 10\% del estimado y 95\% de confianza, se obtuvo que el tamaño mínimo de la muestra debiera ser 369 personas.

Los criterios de inclusión consideraron ser mujer de 40 a 59 años de edad y salud normal, definida por el National Center for Health Statistics como compatible con el desempeño de las actividades rutinarias 9 . Se excluyó del estudio las mujeres con déficit mental que les impidiera entender el cuestionario o padecimientos psíquicos o físicos que les dificulten el desarrollo de la actividad sexual.

Se evaluó la función sexual femenina en mujeres de edad media mediante el cuestionario "Índice de Función Sexual Femenina" (IFSF), desarrollado por Rosen y validado al español (Cronbach $>70 \%)^{8}$. Este cuestionario consta de 19 preguntas y se agrupa en seis dominios: deseo, excitación, lubricación, orgasmo, satisfacción y dolor; cada pregunta tiene 5 ó 6 alternativas de respuesta, con un puntaje que varía de 0 a 5 en cada pregunta. El puntaje de cada dominio se multiplica por un factor y el resultado final es la suma aritmética de los dominios, a mayor puntaje mejor sexualidad. El punto de corte para definir disfunción sexual es un puntaje menor a 26,55 puntos $^{10}$.

Se consignó además la edad, años de estudio, estado civil, presencia o no de menstruaciones, paridad, cirugía ginecológica, uso de terapia hormonal de reemplazo, antecedente de violación, asistencia regular a la iglesia y autopercepción de salud.

El protocolo del estudio fue aprobado por el Comité de Ética de la Fundación PROSAM y previa aplicación de la encuesta se solicitó el consentimiento informado a cada paciente.

Estadística. Se empleó el programa Epi-Info versión 6.04 (Centers for Disease Control, USA; OMS, Suiza). La comparación entre los grupos fue evaluada con ANOVA o con Kruskall-Wallis, según homogeneidad de la varianza medida con la prueba de Bartlet. Los resultados se expresaron como promedio \pm desviación estándar, considerándose significación estadística valores de $\mathrm{p}$ $<0,05$. Para la evaluación simultánea de la influencia de diferentes variables sobre la función sexual femenina se realizó un análisis de regresión logística con el programa Epi-Info 2000.

\section{RESUlTADOS}

Se solicitó a 412 mujeres que contestaran la encuesta "Índice de Función Sexual Femenina", 42 (10\%) rechazaron participar en ella. Se presentan los resultados de 370 mujeres.

En la Tabla 1 se muestran algunas características biosociodemográficas de estas mujeres como un solo grupo; y, separadas por actividad sexual. La edad media de todas las encuestadas fue de 49,3 \pm 5,8 años, con una escolaridad promedio de 10,8 \pm 3,4 años y $68,1 \%$ de ellas tenía pareja estable. La paridad promedio era de $2,8 \pm 1,5$ niños, $44,3 \%$ eran postmenopáusicas, $6,2 \%$ eran usuarias de terapia hormonal y $95,4 \%$ percibían su salud como normal. Dentro del grupo de las mujeres postmenopáusicas, a cuarenta $(24,4 \%)$ se les había realizado histerectomía, 30 (75,0\% de las histerectomizadas) de ellas con salpingooforectomía bilateral asociada. 
Tabla 1. C aracterización de las mujeres estudiadas, agrupadas según actividad sexual

\begin{tabular}{|lcccc|}
\hline Variables & $\begin{array}{c}\text { Mujeres con } \\
\text { actividad sexual }\end{array}$ & $\begin{array}{c}\text { Mujeres sin } \\
\text { actividad sexual }\end{array}$ & Valor P & Total \\
\hline Total N (\%) & $247(66,8)$ & $123(33,2)$ & & $370(100)$ \\
Edad (años) & $48,1 \pm 5,5$ & $51,7 \pm 5,5$ & 0,0001 & $49,3 \pm 5,8$ \\
Escolaridad (años) & $10,9 \pm 3,2$ & $10,7 \pm 3,8$ & NS & $10,8 \pm 3,4$ \\
Pareja estable N (\%) & $196(79,4)$ & $56(45,5)$ & 0,0001 & $252(68,1)$ \\
Paridad (n' hijos) & $2,8 \pm 1,4$ & $2,9 \pm 1,7$ & NS & $2,8 \pm 1,5$ \\
Postmenopausica N (\%) & $87(35,2)$ & $77(62)$ & 0,0001 & $164(44,3)$ \\
Uso de terapia hormonal N (\%) & $15(6,1)$ & $8(6,5)$ & NS & $23(6,2)$ \\
Percepción salud normal N (\%) & $234(94,7)$ & $119(96,7)$ & NS & $353(95,4)$ \\
\hline
\end{tabular}

Tabla 2. Puntajes del IFSF agrupados por edad en mujeres sexualmente activas ( $\mathrm{n}=\mathbf{2 4 7}$ )

\begin{tabular}{|lccccccc|}
\hline $\begin{array}{l}\text { Edad } \\
\text { (años) }\end{array}$ & Deseo & Excitación & Lubricación & Orgasmo & Satisfacción & Dolor & Total \\
\hline $40-44$ & $4,11 \pm 1,29$ & $4,68 \pm 1,07$ & $5,05 \pm 0,93$ & $5,02 \pm 0,95$ & $4,99 \pm 0,95$ & $4,72 \pm 1,07$ & $28,57 \pm 4,63$ \\
$45-49$ & $3,91 \pm 1,02$ & $4,59 \pm 0,90$ & $5,10 \pm 0,97$ & $4,89 \pm 1,00$ & $4,85 \pm 1,11$ & $4,94 \pm 1,15$ & $28,27 \pm 4,25$ \\
$50-54$ & $4,06 \pm 1,26$ & $4,61 \pm 1,16$ & $4,78 \pm 1,29$ & $4,70 \pm 1,34$ & $5,11 \pm 0,98$ & $5,02 \pm 1,09$ & $28,28 \pm 5,53$ \\
$55-59$ & $3,57 \pm 1,0707$ & $4,24 \pm 0,98$ & $3,77 \pm 1,24$ & $4,16 \pm 1,40$ & $4,41 \pm 1,41$ & $4,28 \pm 1,47$ & $24,43 \pm 5,53$ \\
Total & $3,95 \pm 1,21$ & $4,57 \pm 1,03$ & $4,81 \pm 1,16$ & $4,77 \pm 1,16$ & $4,89 \pm 1,09$ & $4,78 \pm 1,18$ & $27,79 \pm 5,06$ \\
p $<$ & 0,135 & 0,186 & $0,0001^{*}$ & $0,01^{*}$ & $0,07^{*}$ & 0,01 & 0,0001 \\
\hline
\end{tabular}

Puntajes más altos representan mejores niveles de función sexual para cada dominio.

*Análisis estadístico con Kruskall-Wallis.

Del total de encuestadas, $123(33,2 \%)$ mujeres se encontraban sin actividad sexual; comparadas con las mujeres con actividad sexual, las inactivas tenían significativamente más edad, un porcentaje menor tenía pareja estable y más eran postmenopáusicas.

La Tabla 2 muestra los puntajes del IFSF de las mujeres sexualmente activas agrupados por edad. Se observa en ellas una caída significativa del puntaje total de casi 4 puntos en el grupo de 55 a 59 años, comparada con las más jóvenes $(28,57 \pm$ $4,63$ vs $24,43 \pm 5,53 ; p<0,0001)$. En cuanto a los dominios evaluados por el IFSF, los más afectados por la edad son la lubricación, que cae desde 5,05 $\pm 0,93$ a $3,77 \pm 1,248(\mathrm{p}<0,0001)$; el orgasmo, desde $5,02 \pm 0,95$ a $4,16 \pm 1,40(\mathrm{p}<0.01)$ y el dolor, desde 4,72 $\pm 1,07$ a 4,28 $\pm 1,47(\mathrm{p}<0,01)$.
En la Tabla 3 se observa que la prevalencia de disfunción sexual, score total del IFSF menor de 26,55, aumenta significativamente con la edad,

\section{Tabla 3. D isfunción sexual por edad en mujeres sexualmente activas}

\begin{tabular}{|lc|}
\hline Edad (años) & D isfunción sexual/total (\%) \\
\hline $40-44$ & $26 / 82(31,7)^{*}$ \\
$45-49$ & $19 / 70(27,1)$ \\
$50-54$ & $19 / 58(32,8)$ \\
$55-59$ & $24 / 37(64,9)^{*}$ \\
Total & $88 / 247(35,6)$ \\
\hline
\end{tabular}

${ }^{*} \mathrm{p}<0,0007$ 
observándose que la mayoría $(64,9 \%)$ de las mujeres entre 55 y 59 años presentaban disfunción sexual comparada con $31,7 \%$ de las encuestadas cuyas edades fluctuaban entre 40 y 44 años $(p<0,0007)$.

En la Tabla 4 se aprecia el impacto de diversas variables de salud y socioculturales sobre el riesgo de tener disfunción sexual. De las variables que aumentan el riesgo significativamente destacan la edad superior a 48 años, la paridad mayor de dos hijos y el estado postmenopáusico. No parecen influir el antecedente de violación, el uso de TRH y la histerectomía. Como factor que disminuye el riesgo se encuentra la percepción de salud normal.

Sin embargo, al realizar regresión logística resultan factores de riesgo significativos sólo la edad superior a 48 años, la paridad mayor de dos hijos y la percepción de salud (Tabla 5). La asistencia regular a iglesias fue un factor que tendió a ser protector.

\section{DiSCUSIÓN}

La sexualidad es una parte integral y relevante de la calidad de vida y es alterada por la menopausia ${ }^{2}$. Sin embargo, la función sexual no sólo depende de los niveles estrogénicos, sino que de la interacción de factores biológicos con aspectos sicológicos, sociales y culturales, factores propios de cada mujer y que le dan la característica de ser una cualidad individual ${ }^{11}$.

En este estudio, llama la atención que un tercio de las mujeres evaluadas se encontraba sin actividad sexual, teniendo $45 \%$ de ellas una pareja estable. Dentro de las razones que explican la inactividad sexual en mujeres de edad media, la disfunción sexual explica la mitad de los casos y el restante está dado por similares proporciones por mala relación con la pareja o la falta de ella ${ }^{12}$.

Nuestros resultados muestran un alto porcentaje de mujeres con disfunción sexual en el período del climaterio, llegando a 57\%, cifra cercana a la descrita en otro estudio nacional similar $^{5}$, que describe una prevalencia de $50,2 \%$ de trastornos de la sexualidad en mujeres climatéricas, pero utilizando el test de Laumann, el cual no contempla los dominios lubricación y satisfacción, que sí incluye el IFSF.

Otro estudio chileno, esta vez utilizando el IFSF para evaluar el impacto de la TRH en mujeres

Tabla 4. Riesgo de disfunción sexual en mujeres sexualmente activas (análisis univariado)

\begin{tabular}{|lccr|}
\hline Variables & OR & IC 95\% & Valor $\mathbf{P}$ \\
\hline Edad $>$ 48 años & 2,10 & $1,19-3,71$ & 0,005 \\
Paridad $>2$ hijos & 1,96 & $1,10-3,49$ & 0,01 \\
Post menopáusica & 1,84 & $1,03-3,29$ & 0,02 \\
Antec. histerectomía & 1,28 & $0,48-3,40$ & NS \\
Uso de terapia hormonal & 2,17 & $0,68-7,01$ & NS \\
Antecedente de violación & 0,94 & $0,38-2,29$ & NS \\
Asistir a iglesia & 0,72 & $0,41-1,27$ & NS \\
Educación $>12$ años & 0,65 & $0,37-1,13$ & NS \\
Percepción salud normal & 0,23 & $0,06-0,85$ & 0,014 \\
\hline
\end{tabular}

Tabla 5. Factores de riesgo de disfunción sexual en mujeres sexualmente activas (análisis de regresión logística)

\begin{tabular}{|lccc|}
\hline Factores de riesgo & OR & IC $\mathbf{9 5 \%}$ & Valor P \\
\hline Problemas de salud & 3,98 & $1,14-13,8$ & 0,030 \\
Edad $>48$ años & 1,95 & $1,12-3,39$ & 0,018 \\
Paridad >2 hijos & 1,76 & $1,00-3,09$ & 0,046 \\
Asistir a iglesia & 0,69 & $0,39-1,19$ & 0,18 \\
\hline
\end{tabular}


climatéricas, mostró que el puntaje total se deteriora significativamente con la edad ${ }^{13}$. Sin embargo, a la fecha de publicación de ese estudio no se había aún establecido un puntaje que definiera disfunción sexual y por lo tanto no da cifras de prevalencia de disfunción, sólo indica como cambian los puntajes con la edad. El presente estudio sería el primero que evalúa la prevalencia de trastornos de la sexualidad en mujeres basado en el International Consensus Development Conference on Female Sexual Dysfunctions.

De los factores evaluados en nuestro estudio, la edad resultó ser un factor de riesgo significativo al afectar la sexualidad femenina globalmente, aumentando el riesgo de disfunción sexual casi al doble, probablemente debido a la disminución hormonal secundaria al envejecimiento ${ }^{14,15}$. Estos resultados coinciden con los descritos previamente por Blümel y cols que también muestran una significativa caída de la sexualidad con la edad ${ }^{13}$. Dentro de los diferentes dominios de la sexualidad, los más afectados por el envejecimiento fueron la lubricación, el orgasmo, el dolor y satisfacción, no así el deseo y la excitación. La atrofia genital secundaria a la disminución de estrógenos se relaciona con disfunción orgánica, lo que explicaría que algunos dominios del IFSF que evalúan aspectos orgánicos como la menor lubricación y el aumento del dolor, fueran afectados significativamente con la edad ${ }^{16}$. Un estudio efectuado por Levine y cols ${ }^{17}$ demuestra que mujeres con disfunción sexual tienen 3,8 veces mayor probabilidad de tener atrofia vulvovaginal, que mujeres sin disfunción, esta atrofia podría explicar la alta prevalencia de trastornos de sexualidad encontrada en este estudio. Con respecto al deseo y la excitación, se

\section{REFERENCIAS}

1. Ojanlatva A, Makinen J, Helenius H, Korkeila K, Sundell J, Rautava P. Sexual activity and perceived health among Finnish middle-aged women. Health Qual Life Outcomes 2006; 4: 29.

2. Castelo-Branco C, Blumel Je, araya H, Riquelme R, Castro G, Haya J, Gramegna G. Prevalence of sexual dysfunction in a cohort of middle-aged women: influences of menopause and hormone replacement therapy. J Obstet Gynaecol 2003; 23: 426-30.

3. Nicolosi A, Laumann EO, Glasser DB, Moreira ED JR, Paik A, Gingell C; Global Study of Sexual Attitudes ha postulado que estos dominios de sexualidad se relacionarían al estado mental de la mujer y a los sentimientos hacia la pareja ${ }^{18}$.

Respecto a otros factores relacionados con la sexualidad femenina, la paridad mayor a dos hijos resultó tener un impacto negativo en la sexualidad femenina en nuestro modelo de regresión logística. En la literatura, estudios realizados en gemelas, reportaron que mujeres nulíparas tenían función sexual superior comparada con mujeres genotípicamente idénticas con paridad mayor a 2 hijos $^{19}$.

La menopausia y disfunción sexual se relacionan directamente en diversos estudios ${ }^{20,21}$. El nuestro no encontró relación significativa con la menopausia al realizar el análisis de regresión logística, sin embargo existen otros factores como la edad y la percepción de salud que demostraron ser más determinantes en la disfunción sexual. Una posible explicación de por qué la menopausia no resultó tan significativa como la edad, es que los síntomas que producen sintomatología suficiente para provocar disfunción sexual, según el IFSF, se dan en la etapa tardía de la menopausia, la cual corresponde aproximadamente al rango de 55 y 59 años, que es el segmento etario con los puntajes IFSF más bajos.

En conclusión, este estudio evidencia en nuestra población la alta prevalencia de disfunción sexual durante el período del climaterio y su relación directa con la edad. En este sentido, la evaluación mediante el IFSF ha resultado de gran utilidad para lograr un acercamiento más estandarizado a entender y evaluar el funcionamiento sexual femenino y lograr diferenciar clínicamente cuando se trata de variación individual de la sexualidad o disfunción de la misma.

and Behaviors Investigators' Group. Sexual behavior and sexual dysfunctions after age 40: the global study of sexual attitudes and behaviors. Urology 2004; 64: 991-7.

4. Nazareth I, Boynton P, King M. Problems with sexual function in people attending London general practitioners: cross sectional study. BMJ 2003; 327: 423.

5. Blumel J, Araya H, Riguelme R, Castro G, Sánchez F, Gramegna G. Prevalencia de los trastornos de la sexualidad en mujeres climatéricas. Influencia de la menopausia y de la terapia de reemplazo hormonal. Rev Méd Chile 2002; 130: 131-8. 
6. Basson R, Berman J, Burnett A, Derogatis L, Fergurson D, Fourcroy J ET aL. Report of the International Consensus Development Conference on Female Sexual Dysfunction: definitions and classifications. $J$ Urol 2000; 163: 888-93.

7. Rosen R, Brown C, Heiman J, Leiblum S, Meston C, Shabsigh R et al. The Female Sexual Function Index (FSFI): A multidimensional self-report instrument for the assessment of female sexual function. J Sex Marital Ther 2000; 26: 191-208.

8. Blumel Je, Binfa L, Cataldo L, Carrasco A, izaguirre H, SARRa S. Indice de función sexual femenina: un test para evaluar la sexualidad de la mujer. Rev Chil Obstet Ginecol 2004; 69: 118-25.

9. BRETt KM, ChOng Y. Hormone Replacement Therapy: Knowledge and Use in the States Unites. Hyattsville, Maryland: National Center for Health Statistics, 2001.

10. Wiegel M, Meston C, Rosen R. The female sexual function index (FSFI): cross-validation and development of clinical cutoff scores. I Sex Marital Ther 2005; 31: 1-20.

11. Genazzani AR, Nicolucci A, Campagnoli C, Crosignani P, Nappi C, Serra GB et al. Assessment of the QoL in Italian menopausal women: comparison between HRT users and non-users. Maturitas 2002; 42: $267-$ 80.

12. Blumel Je, Castelo-Branco C, Cancelo MJ. Impairment of sexual activity in middle-aged women in Chile. Menopause 2004; 11: 78-81.
13. Blumel Je, Bravo F, Recavarren M, Sarrá S. Función sexual en mujeres usuarias de terapia de reemplazo hormonal. Rev Méd Chile 2003; 131: 1251-5.

14. Graziottin A, Leiblum SR. Biological and psychosocial pathophysiology of female sexual dysfunction during the menopausal transition. J Sex Med 2005; 2 Suppl 3: 133-45.

15. Nappi RE, Albani F, Valentino V, Polatti F, Chiovato L, Genazzani AR. Aging and sexuality in women. Minerva Ginecol 2007; 59: 287-98.

16. BACHMANn GA. Influence of menopause on sexuality. Int J Fertil Menopausal Stud 1995; 40 Suppl 1: 16-22.

17. Levine KB, Williams RE, Hartmann KE. Vulvovaginal atrophy is strongly associated with female sexual dysfunction among sexually active postmenopausal women. Menopause 2008, 15: 661-6.

18. Basson R, Brotto la, Laan E, Redmond G, Utian WH. Assessment and management of women's sexual dysfunctions: problematic desire and arousal. J Sex Med 2005; 2: 291-300.

19. Botros S, Abramov Y, Miller J, Sand P, Gandhi S, Nickolov A, Goldberg R. Effect of Parity on Sexual Function. Obstet Gynecol 2006; 107: 765-70.

20. Mansfield PK, Koch PB, Voda AM. Midlife women's attributions for their sexual response changes. Health Care Women Int 2000; 21: 543-59.

21. Avis N, Stellato R, Crawford S, Johannes C, Longcope C. Is there an association between menopause status and sexual functioning? Menopause 2000; 7: 297-309. 